\title{
Could AAA screening reduce mortality?
}

A systematic review and meta-analysis has shown that screening the general population of asymptomatic elderly men for abdominal aortic aneurysm (AAA) is associated with a $50 \%$ reduction in AAA-related mortality.

AAA rupture, which is often a fatal event, is commonly the first manifestation of an aneurysm. AAAs of treatable size (30-55 $\mathrm{mm}$ ) are clinically 'silent' in the majority of patients. Screening for AAAs in high-risk populations could, therefore, help to identify these lesions at an early stage, and reduce morbidity and mortality.

From a total of 204 full-text papers identified in a literature search, four randomized controlled trials, which the investigators deemed to be of good or fair quality, were selected for the meta-analysis. In each of these trials (the Multicentre Aneurysm Screening Study [MASS]; the Viborg County, Denmark screening trial; the Chichester, UK screening trial; and the Western Australia screening trial), one-off ultrasonographic screening was investigated in asymptomatic men (or men and women in the Chichester study) aged $\geq 64-65$ years.
The prevalence of AAA was 4.0-7.7\%, and the majority of detected lesions were small $(<40-45 \mathrm{~mm})$. AAAs at risk of rupture $(\geq 55 \mathrm{~mm})$ were detected in only $0.4-0.6 \%$ of trial participants. AAA mortality was significantly reduced in screened men compared with those who did not undergo screening in MASS (HR 0.58, $P \leq 0.05$ at mean follow-up 4.1 years) and the Viborg trial (HR 0.33 , $P \leq 0.05$ at median follow-up 4.3 years); the risk of rupture was also reduced in these studies. AAA screening was not associated with a reduction in all-cause mortality. In the Chichester study, screening of women was not linked with reductions in AAA rupture or mortality. The investigators call for further research, using a "multifactorial risk calculator" to identify individuals for screening.

\section{Alexandra Roberts}

Original article Guirguis-Blake, J. M. et al. Ultrasonography screening for abdominal aortic aneurysms: a systematic evidence review for the US Preventive Services Task Force. Ann. Intern. Med. doi:10.7326/M13-1844 